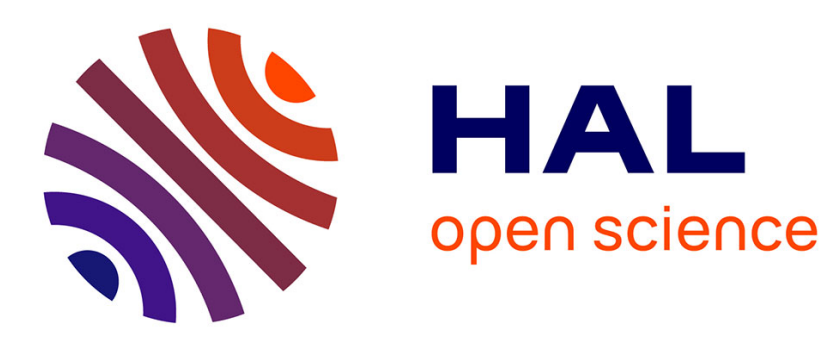

\title{
Multifractal vector fields and stochastic Clifford algebra
}

D Schertzer, Ioulia Tchiguirinskaia

\section{- To cite this version:}

D Schertzer, Ioulia Tchiguirinskaia. Multifractal vector fields and stochastic Clifford algebra. Chaos: An Interdisciplinary Journal of Nonlinear Science, 2015, 25 (12), 10.1063/1.4937364 . hal-01299360

\section{HAL Id: hal-01299360 \\ https://hal-enpc.archives-ouvertes.fr/hal-01299360}

Submitted on 7 Apr 2016

HAL is a multi-disciplinary open access archive for the deposit and dissemination of scientific research documents, whether they are published or not. The documents may come from teaching and research institutions in France or abroad, or from public or private research centers.
L'archive ouverte pluridisciplinaire HAL, est destinée au dépôt et à la diffusion de documents scientifiques de niveau recherche, publiés ou non, émanant des établissements d'enseignement et de recherche français ou étrangers, des laboratoires publics ou privés. 


\section{$\mathrm{AIP} \mid$ chaos \\ An Interdisciplinary Journal of Nonlinear Science}

\section{Multifractal vector fields and stochastic Clifford algebra}

Daniel Schertzer and loulia Tchiguirinskaia

Citation: Chaos 25, 123127 (2015); doi: 10.1063/1.4937364

View online: http://dx.doi.org/10.1063/1.4937364

View Table of Contents: http://scitation.aip.org/content/aip/journal/chaos/25/12?ver=pdfcov

Published by the AIP Publishing

\section{Articles you may be interested in}

Designing a stochastic genetic switch by coupling chaos and bistability

Chaos 25, 113112 (2015); 10.1063/1.4936087

Stochastic bifurcation and fractal and chaos control of a giant magnetostrictive film-shape memory alloy composite cantilever plate subjected to in-plane harmonic and stochastic excitation

J. Appl. Phys. 115, 17E527 (2014); 10.1063/1.4868182

Multifractal transport in the standard map

AIP Conf. Proc. 1444, 476 (2012); 10.1063/1.4715481

Lagrangian stochastic modeling of anomalous diffusion in two-dimensional turbulence

Phys. Fluids 14, 1442 (2002); 10.1063/1.1456063

Monofractal and multifractal approaches to complex biomedical signals

AIP Conf. Proc. 502, 133 (2000); 10.1063/1.1302377

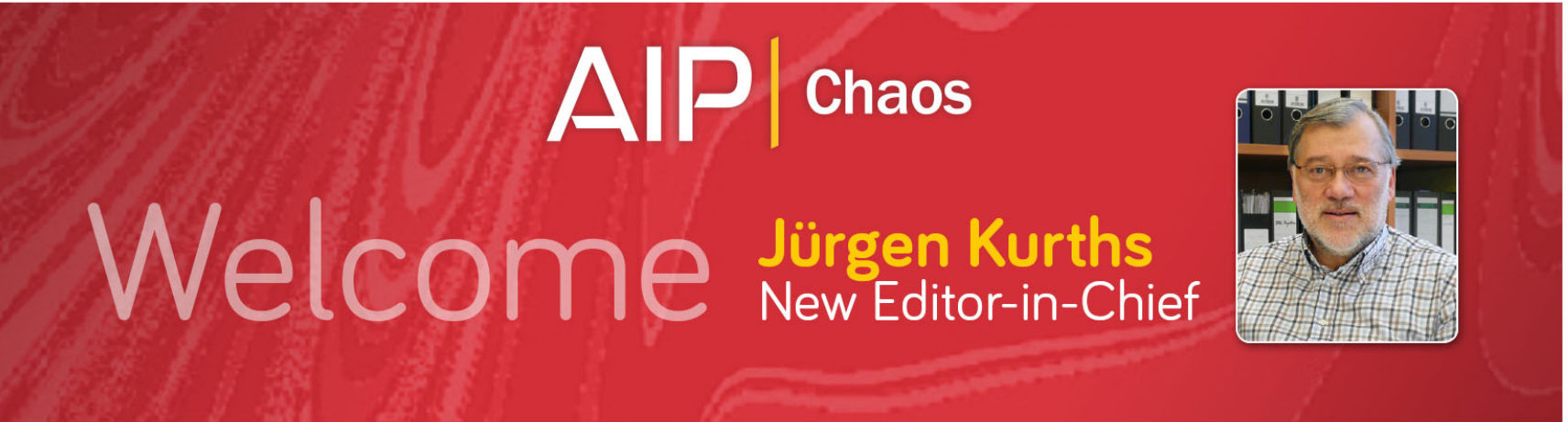




\title{
Multifractal vector fields and stochastic Clifford algebra
}

\author{
Daniel Schertzer ${ }^{a)}$ and loulia Tchiguirinskaia ${ }^{\text {b) }}$ \\ University Paris-Est, Ecole des Ponts ParisTech, Hydrology Meteorology and Complexity (HM\&Co), \\ Marne-la-Vallée, France
}

(Received 13 July 2015; accepted 23 November 2015; published online 31 December 2015)

\begin{abstract}
In the mid 1980s, the development of multifractal concepts and techniques was an important breakthrough for complex system analysis and simulation, in particular, in turbulence and hydrology. Multifractals indeed aimed to track and simulate the scaling singularities of the underlying equations instead of relying on numerical, scale truncated simulations or on simplified conceptual models. However, this development has been rather limited to deal with scalar fields, whereas most of the fields of interest are vector-valued or even manifold-valued. We show in this paper that the combination of stable Lévy processes with Clifford algebra is a good candidate to bridge up the present gap between theory and applications. We show that it indeed defines a convenient framework to generate multifractal vector fields, possibly multifractal manifold-valued fields, based on a few fundamental and complementary properties of Lévy processes and Clifford algebra. In particular, the vector structure of these algebra is much more tractable than the manifold structure of symmetry groups while the Lévy stability grants a given statistical universality. (C) 2015 Author(s). All article content, except where otherwise noted, is licensed under a Creative Commons Attribution 3.0 Unported License. [http://dx.doi.org/10.1063/1.4937364]
\end{abstract}

The multifractal framework is very convenient to analyze and simulate extremely variable fields over a wide range of space-time scales. However, it has been mostly developed for scalar fields and is therefore not yet suitable for many applications. This paper demonstrates that combining two special, but wide enough classes of stochastic processes ("stable Levy processes") and algebra ("Clifford algebra") provides a convenient mathematical and physical framework for vector multifractals and possibly manifold valued multifractals, i.e., for multifractal fields whose values belong to a manifold (e.g., a flow on this manifold), therefore for a wide range of complex systems. This paper does not intend to be reserved for specialists and therefore provides pedagogical introductions to concepts that are used.

\section{INTRODUCTION}

\section{A. What is at stake?}

Multifractals are space-time fields that have structures at all scales. Scalar valued multifractal fields are already a broad generalization of the (geometrical) fractals, because they are no longer limited to be binary functions, like the set indicator function of a fractal set ( $=0$ or 1 ), but have a continuum of levels of activity, each of them supported by a given fractal set, hence the term "multifractal." The loose, but appealing, expression "activity" is precisely quantified by the small scale

Note: Paper submitted as part of the July, 2015 focus issue: PHYSICS OF SCALING AND SELF-SIMILARITY IN HYDROLOGIC DYNAMICS, HYDRODYNAMICS AND CLIMATE (Guest Editors: M. L. Kavvas, R. S. Govindaraju, and U. Lall)

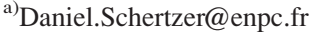

${ }^{\text {b) } I o u l i a . T c h i g u i r i n s k a i a @ e n p c . f r}$ singular behavior of the field, e.g., that of the (scalar) energy flux density for hydrodynamical turbulence, whose challenging intermittency largely motivated the development of multifractals. ${ }^{1-5}$ Multifractals were thus widely used to analyse and simulate the extreme variability of numerous fields, ranging from high-energy physics ${ }^{6,7}$ to climate ${ }^{8-11}$ including financial fluctuations, ${ }^{12,13}$ as well as galaxy distributions. ${ }^{14,15}$

However, multifractal approaches were mostly developed for scalar valued fields, whereas the fields of interest, e.g., the velocity for turbulence and the discharge for hydrology, are generally vector fields. Furthermore, a convenient (statistical) rotation invariance is usually pre-supposed, whereas this is rarely relevant for real systems, which have often preferred directions. Both gaps between theories and applications have prevented many developments. This paper is devoted to overcome them by showing that the vector nature of the domain and/or the codomain of a field-respectively the set over which it is defined and the set onto it is valued-is not a fundamental obstacle, although it introduces some technical difficulties, which turn nevertheless to be very stimulating.

The property to have structures at all scales is trivially scale invariant since it does not depend on the scale of observation. It either does not depend on the dimension of the codomain. A multifractal field can therefore be defined as being invariant for a given scale transform, which is thus a symmetry of this field. Such a field is said to be scale invariant or a scaling for short. We will give a general, precise definition of this invariance and the corresponding scale transform that could be either deterministic or stochastic (e.g., involves only equality in probability distribution or other statistical equivalences), isotropic or not. In this framework, multifractals are therefore both quite general and fundamental. Indeed, not only are symmetry principles the building blocks of physics and many other disciplines ${ }^{16,17}$ but also scale symmetry is an 
element of the extended Galilean invariance. Unfortunately, attention in mechanics, especially in point mechanics, has been initially focused on the Galilean transformation between two reference frames that differ only by a constant relative velocity or by a given rotation that define the pure Galilean group. But with extended bodies, therefore continuous mechanics, it broadened to other transforms such as scale dilations. In particular, Sedov ${ }^{18}$ demonstrated further to the $\Pi$ theorem of Buckimgham ${ }^{19-21}$ the key role of the latter in fluid mechanics, including for many practical applications. More recently, this was related to Lie groups of symmetry. ${ }^{22-24}$ On the other hand, another approach was based on stochastic differential calculus. ${ }^{25-27}$ In a given way, this paper combines both approaches with the help of the complementary properties of stable Lévy processes and Clifford algebra. Both have particularly important generic properties respectively as stochastic processes and Lie algebra.

\section{B. How is organized this paper?}

This paper aims to be self-consistent and pedagogical as much as possible, whereas at the same time it combines two different types of approaches and techniques: multifractals generated by stable Lévy processes and the Lie algebra of symmetry generators. We hope that the following organization of the paper will help to overcome the resulting intrinsic complexity (Fig. 1).

Section II recalls the necessity for scale invariance to handle generalized scales ${ }^{28}$ instead of classical scales, i.e., those equivalent to the Euclidean metrics. This is introduced in a geometric manner on the domain of a given field and enables to introduce the concept of generalized scale transform that is a building block of other sections. For instance, Sec. III introduces the notion of pullback and push-forward transforms of a field due to the application of the generalized scale transform on its domain. It therefore enables us to go from a geometrical concept to an analytical one: how functions and measures are respectively transformed? This yields (Sec. IV) a very general definition of a multifractal field with the help of generalized scale transforms on both its domain and codomain.

Before attacking their generalization to higher dimensions, the main features of scalar-valued multifractal fields are summarized in Sec. V after a terse recapitulation of stable Lévy variables (Sec. V A) that can be skipped by those who are familiar with them. However, the following

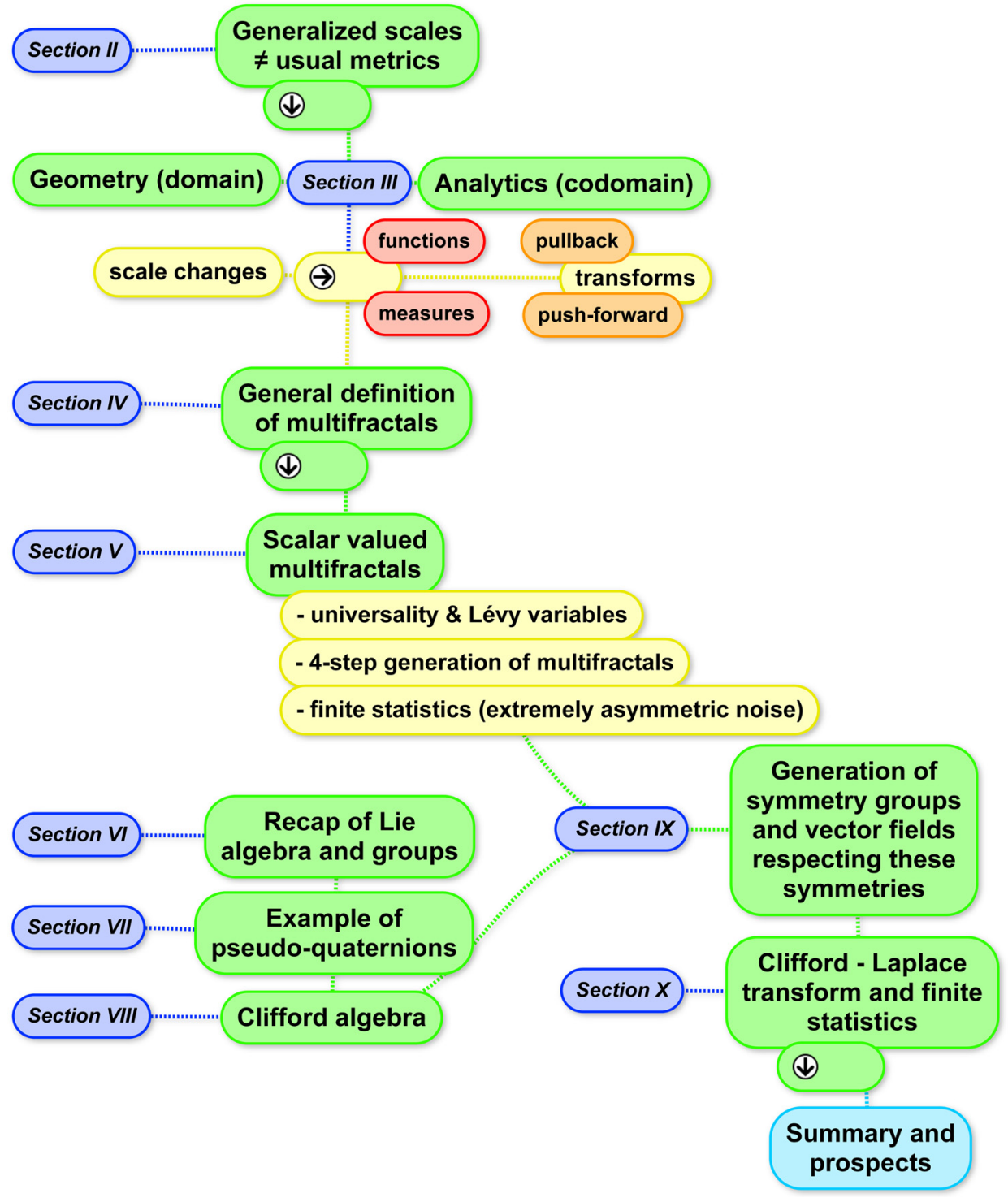

FIG. 1. This figure schematically displays the organisation of our paper and the interplay between the main concepts. 
subsection (Sec. V B) first discusses the nonclassical case of extremely asymmetrical stable Lévy variables, because the Lévy generators are necessarily of this type. The latter yield the particular, but already large, case of universal multifrac$\operatorname{tals}^{29,30}$ whose 4-step generation is then presented.

Section VI is a transition to Lie algebra and groups, which can be overlooked by readers familiar with these concepts. It is rather the same with Sec. VII that presents a simple example of a Lie algebra, also of a Clifford algebra, although the latter property is not so well known. General properties of Clifford algebra are presented and discussed in Sec. VIII. We have then the necessary tools to address in Sec. IX the main goal of this paper: to generate both symmetry groups and vector fields respecting these symmetries. They fundamentally correspond to integrate a vector field into a flow, ${ }^{31}$ but in a rather uncommon framework: the vector field is already a fractionally integrated Lévy white noise vector, whereas there had been only rather limited mathematical works ${ }^{32,33}$ on the classical integration of Lévy white noise vectors into a flow. Nevertheless, a rigorous condition for the existence of these fields and symmetries is obtained with the help of a Laplace-Clifford transform in Sec. X that also summarizes the results and draw prospects.

\section{GENERALIZED SCALE INVARIANCE (GSI)}

The ubiquitous anisotropy of geophysical fields and patterns first requires to generalize the concept of scale well beyond the Euclidean metrics, which had surprisingly remained a building block of the (non Euclidean) Fractal Geometry of Nature. ${ }^{34}$ In fact, the concept of GSI was actually developed to account for the strong scaling stratification of the atmosphere ${ }^{28,35}$ that breaks the rotational symmetry. The corresponding GSI model has gained increasing empirical confirmation with the help of various atmospheric measurements. ${ }^{36-40}$ More recently, ${ }^{41}$ it was theoretically used to go beyond the quasi-geostrophic approximation limitations. For illustration purpose, Fig. 2 displays such generalized scales, which no longer correspond to self-similar spheres, and that we now define before discussing more this figure.

Let $\varepsilon$ be a field with domain $X$ and codomain $Y$, which could be in a general manner manifolds but are presently assumed to be vector spaces for notation simplicity sake
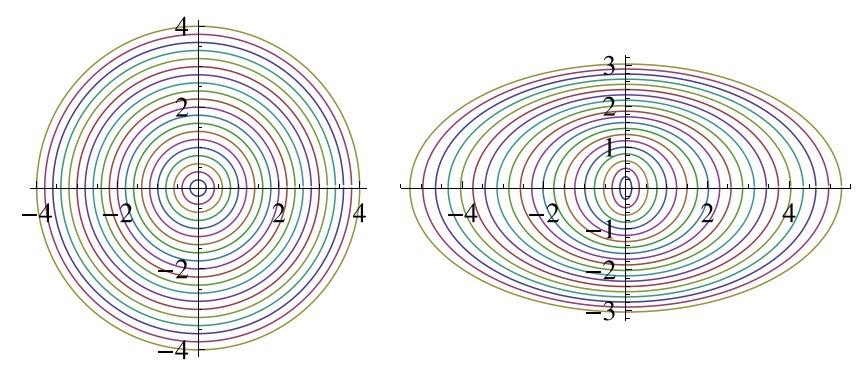

FIG. 2. Contours of $2 \mathrm{D}$ balls $B_{\lambda}=T_{\lambda}\left(B_{1}\right)$, where $B_{1}$ is the unit circle, for scale ratios $\lambda \in(1 / 4,5)$ by steps of $\delta(1 / \lambda)=0.2$ : (a, on the left) for an isotropic scale transform $T_{\lambda} x=x / \lambda$, i.e., self-similar balls of the Euclidean metrics (b, on the right) generalized scales in a vertical plane to analyse and simulate stratified dynamics: the balls $B_{\lambda}$ 's are no longer self-similar circles, but self-affine ellipses with $T_{\lambda} x=x / \lambda^{G}$, where $G$ is diagonal with eigenvalues $d+c=1.2$ and $d-c=0.8$.

$$
X \stackrel{\varepsilon}{\rightarrow} Y
$$

that is, $\varepsilon$ belongs to a given functional space $S(X, Y)$. An isotropic scale transformation is merely defined with the help of usual (isotropic) contractions/dilations $T_{\lambda}$ of the domain $X$ for any positive scale ratio $\lambda(\lambda>1$ for a contraction, $0<\lambda<1$ for a dilation)

$$
x \mapsto T_{\lambda} x=x / \lambda
$$

that satisfy the scalability relation for any classical norm $|$.

$$
\left|T_{\lambda} x\right|=|x| / \lambda \text {. }
$$

These properties can be generalized in the following manner: $T_{\lambda}$ is a generalized contraction of $X$, if it is a oneparameter (semi-) group for the positive scale ratio $\lambda(\lambda \geq 1$ for a semi- group), i.e.,

$$
T_{\lambda \lambda^{\prime}}=T_{\lambda^{\prime}} \circ T_{\lambda}
$$

and admits a generalized scale denoted $\|x\|$ (to distinguish it from a classical norm $|x|$ ), which satisfies the following three properties $^{42}$ further to that of being non-negative

(1) non degeneracy

$$
\|x\|=0 \Rightarrow x=0
$$

(2) scalability

$$
\left\|T_{\lambda} x\right\|=\|x\| / \lambda
$$

(3) ball embedding

$$
\forall L \in R^{+}, \lambda^{\prime} \geq \lambda \geq 1: B_{L / \lambda^{\prime}} \subset B_{L / \lambda},
$$

where the (closed) balls $B_{l}$ are defined with the help of the generalized scale $\|$.

$$
B_{l}=\{x \mid\|x\| \leq l\}
$$

the corresponding open balls, which define the topology, are obtained with the help of a strict inequality.

With respect to the corresponding required properties of a norm, the nondegeneracy property (1) is unchanged, the scalability property (2) is formally similar (but generalizes Eq. (3) to any anisotropic generalized contraction $T_{\lambda}$ ), whereas the condition of embedding of the balls defined by the generalized scale (3) is weaker than the triangular inequality. Obviously, a classical norm $|x|$ is the scale associated to the isotropic contraction (Eq. (2)). A rather trivial example of generalized scale is obtained in the framework of linear GSI, where the scale transform is defined by a given matrix $\mathrm{G}$ by

$$
T_{\lambda}=\lambda^{G} \equiv \exp (\log (\lambda) G) .
$$

Let us consider the case of a diagonalizable matrix $G$ with a positive spectrum $\operatorname{Spec}(G)=\left\{\nu_{i}\right\}$ and corresponding eigenvectors $\left\{e^{i}\right\}$, which corresponds to the self-affine case. ${ }^{43} \mathrm{~A}$ generalized scale is merely defined as follows for any positive $\alpha$ :

$$
\left\|\sum_{i} x_{i} e^{i}\right\|=\left(\sum_{i}\left\|x_{i} e^{i}\right\|^{\alpha}\right)^{1 / \alpha} ;|| x_{i} e^{i} \|=\left|x_{i}\right|^{1 / \nu_{i}}\left|e^{i}\right| .
$$


This example points out that, like for usual norms, there are many possible definitions of generalized scales that could share some common properties. It is therefore important to have a similar definition of equivalence, i.e., the generalized scales $\|.\|_{1}$ and $\|.\|_{2}$ are equivalent if there are two constants $A$ and $B$ such that

$$
\forall x \in X: A\|x\|_{1} \leq\|x\|_{2} \leq B\|x\|_{1} .
$$

In what follows, the condition of "fractional scalability" will be useful, i.e., a generalized scale $\| .||$ being defined in relation with a given generalized scale transform $T_{\lambda}$, other transformation $\tilde{T}_{\lambda}$ may satisfy a scalability condition involving an exponent $H \neq 1$

$$
\left\|\tilde{T}_{\lambda} x\right\|=\|x\| / \lambda^{H} .
$$

\section{PULLBACK AND PUSH-FORWARD TRANSFORMS}

It is very convenient to use the concept of a pullback transform of a function and that of a push-forward transform of a (mathematical) measure.

The pullback transform (or "composition operator" ${ }^{44}$ ) by a given space transform is so general that it is often passed over without mentioning it. It corresponds to a straightforward generalization to (infinite dimensional) functional spaces of the (contravariant) change of coordinates of (finite dimensional) vector spaces (see Fig. 3 for illustration). Here, we are interested by the pullback $T_{\lambda}^{*}$ generated by a generalized contraction/dilation $T_{\lambda}$

$$
\forall \varphi \in S(X, Y), \forall x \in X: T_{\lambda}^{*}(\varphi)(x)=\varphi\left(T_{\lambda} x\right) .
$$

The composed function $u\left(T_{\lambda}\right)$ indeed pulls back the field $u$ from the coordinates $y=T_{\lambda}(x)$ to the old coordinates $x$, with the "change of coordinates" $y=T_{\lambda}(x)$.

The push-forward transform $T_{*} \lambda$ is obtained by duality considering measures $\mu^{\prime}$ s $\in S^{\prime}(X, Y)$, i.e., linear transforms over the functional space $S(X, Y)$ of the test functions $\varphi$ (linear forms when the test functions $\varphi$ 's are scalar valued)

$$
\forall \mu \in S^{\prime}(X, Y), \forall \varphi \in S(X, Y): \int T_{\lambda}^{*}(\varphi) d \mu=\int \varphi d\left(T_{* \lambda} \mu\right) .
$$

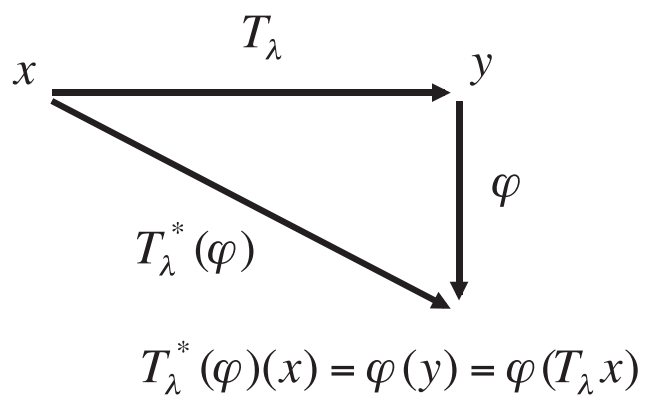

FIG. 3. Scheme of the "pullback" $T_{\lambda}^{*}$ that pulls back the field $u$ from the coordinates $y$ to $x$ with the help of the (anisotropic) space contraction/dilation $T_{\lambda}$.
$T_{*} \lambda$ indeed pushes forward the measure $\mu$ from the coordinates $x$ to the new coordinate $y=T_{\lambda}(x)$ and generalizes the covariant coordinate transform of the (finite dimensional) dual vector spaces. Because multifractals are often measures, rather than pointwise functions, the push forward transform is in fact more relevant than the pullback. In fact, we will consider linear transforms of multifractals, therefore implicitly considering transforms acting on bidual spaces.

\section{A GENERALIZED DEFINITION OF MULTIFRACTALS}

We now have the means to define a multifractal field as an invariant of a given symmetry $S_{\lambda}$

$$
S_{\lambda} \varepsilon=\varepsilon,
$$

where $S_{\lambda}$ results from the composition of a generalized compression/dilation of the domain and a generalized dilation/ compression (possibility with a factional scalability condition, Eq. (12)). Loosely speaking, the generalized scale transform of the domain is compensated by a generalized scale transform of the codomain. In the case of a multifractal function $\varphi$, it corresponds to

$$
S_{\lambda}=\tilde{T}_{\lambda}^{-1} \circ T_{\lambda}^{*},
$$

whereas for a multifractal measure $\mu$, it corresponds to

$$
S_{\lambda}=\tilde{T}_{\lambda}^{-1} \circ T_{* \lambda} .
$$

In a general manner, the equality sign in these equations is not a deterministic one, but a statistical one, e.g., almost surely equality or only equality in distribution. This is precisely the case for the classical mono/uni scaling ${ }^{45}$ for the increments of an additive process

$$
\tilde{T}_{\lambda}=\lambda^{H},
$$

where $H$ is a given (scalar) exponent. This behavior was empirically uncovered in hydrology by Hurst, ${ }^{46,47}$ hence the letter $H$ for this exponent. It is exhibited by fractional Brownian motions. ${ }^{48}$ There is a similar behavior for a fractal/Hausdorff measure of dimension $D$ when applied to a fractal set of dimension $D^{\prime}$. The corresponding scaling exponent is the fractal codimension $C=D-D^{\prime}$. However, the breakthrough of multifractals was to abandon the unicity of the exponent $H$ that is no longer relevant for multiplicative processes. They indeed generate in a very general manner an infinite hierarchy of "singularities" $\gamma$ 's

$$
\tilde{T}_{\lambda}=\lambda^{\gamma}=\exp \left(\Gamma_{\lambda}\right)
$$

where $\Gamma_{\lambda}$ is the stochastic generator of the group $\tilde{T}_{\lambda}$. Equation (19) together with the multiplicative group property of $\tilde{T}_{\lambda}$ (similar to that of $T_{\lambda}$, Eq. (4)) imply an additive group property for $\Gamma_{\lambda}$. This Equation (19) also yields a multiscaling relation for all (finite) statistical moments of $\tilde{T}_{\lambda}$ with the help of the first and second (Laplace) characteristic functions $Z_{\lambda}(q)$ and $K_{\lambda}(q)$ of the generator $\Gamma_{\lambda}$ (where $E[$.] denotes the mathematical expectation) 


$$
E\left[\tilde{T}_{\lambda}^{q}\right]=E\left[\exp \left(q \Gamma_{\lambda}\right)\right]=Z_{\lambda}(q)=\exp \left(K_{\lambda}(q)\right) \approx \lambda^{K(q)}
$$

as soon as $K_{\lambda}(q)$ has a $\log (\lambda)$ divergence

$$
K_{\lambda}(q)=\log \left(\left\langle\exp \left(q \Gamma_{\lambda}\right)\right\rangle\right) \approx \log (\lambda) K(q)
$$

$K(q)$ defines the multi-scaling behavior of the moments $E\left[\tilde{T}_{\lambda}^{q}\right]$ and is therefore called the scaling moment function.

\section{SCALAR-VALUED UNIVERSAL MULTIFRACTALS}

\section{A. Fundamental properties of Lévy stable variables}

A particular case of multifractal generators, which is already general enough, corresponds to stable Lévy generators. Recall that a random variable $X$ is said to be a Levy stable variable, ${ }^{49-52}$ if and only if it is stable under renormalized sums, i.e., it is a fixed point with the rescaling factor $a(n)$ and centering term $b(n)$, of any $n$ of its independent realizations $X_{i},(i=1, n)$. This corresponds to ( ${ }^{d}$ denotes equality in distribution)

$$
\forall n \in N, \exists a(n), b(n) \in R: \sum_{i=1}^{n} X_{i}={ }^{d} a(n) X+b(n) .
$$

$X$ is said to be strictly stable, according to Feller's terminology, when the centering term $b(n)$ is 0 . Furthermore, any Levy stable variable $X$ is attractive for renormalized sum of independent realizations $Y_{i}(i=1, n)$

$$
\lim _{n \rightarrow \infty} \frac{\sum_{i=1}^{n} Y_{i}-b(n)}{a(n)}={ }^{d} X
$$

of a random variable $Y$ having similar distribution tails, i.e., power-law tail whose exponent is the Lévy stability index $\alpha \in[0,2]$

$$
\forall s \gg 1: \operatorname{Pr}(|X|>s) \approx s^{-\alpha}
$$

whereas its inverse $1 / \alpha$ is the generator of the multiplicative group of the renormalizing factor $a(n)$

$$
a(n)=n^{1 / \alpha}
$$

As a consequence of the stability under renormalized sums, the second Fourier characteristic function

$$
K_{F}(q)=\log (E[\exp (i q X)])
$$

of a Levy stable variable $X$ is of the form

$$
K_{F}(q)=i m q-D|q|^{\alpha}\left(1-i \beta \frac{q}{|q|} \omega(q, \alpha)\right) .
$$

where $D$ is the scale parameter, $\beta$ is the skewness parameter, $m$ is the centering term, and the prefactor $\omega$ is defined as follows:

$\alpha \neq 1: \omega(q, \alpha) \equiv \omega(\alpha)=\tan \frac{\pi \alpha}{2} ; \alpha=1: \omega(q, \alpha)=\frac{\pi}{2} \log |q|$.
The Gaussian case $\alpha=2$ is necessarily symmetric because $\omega(2)=0$ and the skewness value $\beta$ is therefore undefined, but rather corresponds to 0 .

\section{B. Lévy stable generators}

Due to the aforementioned properties of stable Lévy variables, the stable Lévy generators generate universal multifractals having the same properties of stability and attractivity for renormalized products. ${ }^{6,29,30,53}$ However, there is an important technical problem due to the fact that the scaling moment function $K(q)$ of $\tilde{T}_{\lambda}$ (Eq. (20)) corresponds to the second Laplace characteristic function of its generator $\Gamma_{\lambda}$. Contrary to the Fourier characteristic function $K_{F}(q)$ (Eq. (27)), the Laplace characteristic function $K(q)$

$$
K(q)=\log (E[\exp (q X)])
$$

does not exist for any Levy stable variable $X$. Indeed, $K(q)$ is finite for non negative moment orders $q$ 's only for fully asymmetrical Lévy stable variables $(\beta=-1)$, i.e., they have a probability falloff power-law only for negative values

$$
\forall s \gg 0: \operatorname{Pr}(X<-s) \approx s^{\alpha} \text {. }
$$

On the contrary, the probability of positive values has a fast falloff. The existence of such a constraint is a not at all a mathematical surprise, but usual for Laplace transforms with respect to Fourier transforms. The present physical reason is that the exponentiation of extreme negative fluctuations only yields extremely low values, whereas extreme positive fluctuations yield much higher fluctuations and therefore divergence of the characteristic function. This is confirmed by the following inequality:

$$
\forall n \in N, \forall X, q \geq 0: \exp (q X) \geq(q X)^{n} / n !
$$

which shows that $K(q)$ cannot be finite as soon a given $n^{\text {th }}$ order moment of the positive fluctuations of the generator is infinite. This in turn requires that the falloff of the probability of the generator is steeper than any power law, because the existence of a finite power law exponent $\alpha$ means that all statistical moments of orders $q \geq \alpha$ are infinite.

The second Laplace characteristic function is of the form

$$
q \geq 0: K(q)=m q+D \operatorname{sign}(\alpha-1) q^{\alpha} ; q<0: K(q)=\infty
$$

with the same meaning for the parameters $m$ and $D$ as for the Fourier characteristic function. Surprisingly, the Laplace characteristic function has been scarcely used and almost exclusively for the case ${ }^{51} \alpha<1$, where the probability is one sided, i.e., stable Lévy variables having an upper bound.

With the help of Eq. (32), the scaling moment function $K(q)$ of the conservative $(K(1)=0)$ universal multifractals can be written under the following form: ${ }^{29}$

$$
q \geq 0: K(q)=\frac{C_{1}}{\alpha-1}\left(q^{\alpha}-q\right) ; q<0: K(q)=\infty
$$


with the help of two parameters $C_{1}$ and $\alpha$ that have strong physical meanings

- $C_{1}$ is the codimension of the mean field: it measures the mean intermittency (or the intermittency of the mean field). It defines the uni/mono fractal field approximation in the neighborhood of the mean field: $C_{1}=d K(q) /\left.d q\right|_{q=1}$. $C_{1}$ measures the amplitude of the generator fluctuations, in particular, $C_{1}=0$ for an homogeneous field.

- $\alpha$ is the multifractality index of the field and the Lévy stability index of the generator with the aforementioned properties (Eqs. (24) and (25)): it measures the variation of the intermittency in the neighborhood of the mean. It is precisely related to the curvature of $K(q): \alpha=d^{2} K(q) /\left.C_{1} d q^{2}\right|_{q=1}$.

Generators having these properties, are obtained by fractional integration of extremely asymmetric Lévy white noises $\gamma_{0}^{(\alpha)}$ of stability index $\alpha$

$$
\begin{aligned}
\Gamma_{\lambda}(x)= & \left|\frac{\operatorname{var}(\alpha)}{m_{D-1}\left(\partial B_{L}(x)\right)}\right|^{1 / \alpha} \int_{B_{L}(x) B_{L / \lambda}(x)} G\left(x-x^{\prime}\right) d \gamma_{0}^{(\alpha)} \\
& -\operatorname{var}(\alpha) \log (\lambda) ; \operatorname{var}(\alpha)=\frac{C_{1}}{\alpha-1},
\end{aligned}
$$

where $\left.B_{L}(x)\right)$ denotes a ball of radius $L$ of dimension $D$ and centered in $x, m_{D-1}\left(\partial B_{L}(x)\right)$ the (D1)-dimensional Lebesgue measure of its (hyper-)surface $\partial B_{L}(x), \operatorname{var}(\alpha)$ corresponds to a generalization of the (quadratic) variation of a (semi-) martingale. ${ }^{54} G(x)$ is the Green function of a fractional Laplace operator $\left(\Delta=\nabla^{2}\right)$

$$
-\left(-\Delta_{x}\right)^{D / 2 \alpha^{\prime}} G\left(x-x^{\prime}\right)=\delta\left(x-x^{\prime}\right) ; G(x) \propto|x|^{-D / \alpha},
$$

where $1 / \alpha+1 / \alpha^{\prime}=1$.

\section{Universal multifractals}

Multiplying the exponential of the generator $\Gamma_{\lambda}$ by the homogeneous (large scale) field $\varepsilon_{1}$, which could be taken as unity without loss of generality, yields a highly inhomogeneous flux $\varepsilon_{\lambda}$. Nevertheless, $\varepsilon_{\lambda}$ is conservative $\left(\forall \lambda, E\left[\varepsilon_{\lambda}\right]=E\left[\varepsilon_{1}\right]\right)$ and more generally is a martingale (the conditional expectation $E_{\lambda}\left[\varepsilon_{\Lambda}\right]$ at resolution $\lambda<\Lambda$ of $\varepsilon_{\Lambda}$ is simply $\varepsilon_{\lambda}$ ). Finally, we can obtain a non-conservative field $v_{\lambda}$, like the velocity field, by fractionally integrating ${ }^{55}$ a given power $a$ th of $\varepsilon_{\lambda}$, i.e., $v_{\lambda}$ is the solution of a fractional diffusion equation, similar to Eq. (35), but of order $\mathrm{H}$ instead of $D 2 / \alpha^{\prime}$ forced by $f_{R}=\varepsilon_{\lambda}^{a}$

$$
v_{\lambda}(x) \propto \int_{B_{L}(x) \backslash B_{L / \lambda}(x)} G_{R}\left(x-x^{\prime}\right) f_{R}\left(x^{\prime}\right) d^{D} x^{\prime} ; \quad f_{R}(x)=\varepsilon^{a}(x)
$$

with

$$
-\left(-\Delta_{x}\right)^{H / 2} G_{R}\left(x-x^{\prime}\right)=\delta\left(x-x^{\prime}\right) ; \quad G_{R}(x) \propto|x|^{-(D-H)} .
$$

Figures 4 and 5 display the time series of the aforementioned steps to obtain a 1D scalar-valued universal multifractal with parameters $C_{1}=0.2, a=1, H=1 / 9$ and respectively for $\alpha=2$ and $\alpha=1.2$

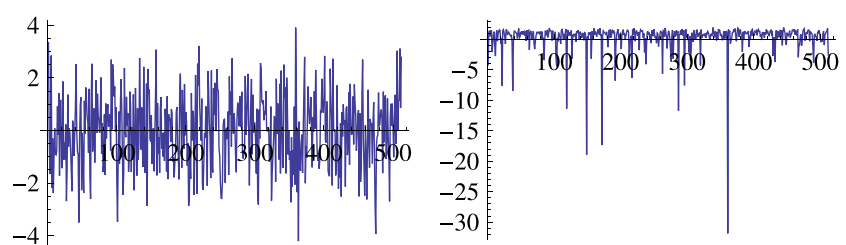

FIG. 4. Examples of white noises $\gamma_{0}^{(\alpha)}$ with $\lambda=512$ (the horizontal axis is the time $t \in(0, \lambda)$ ) admitting a finite second Laplace characteristic function $K(q)$ for $q \geq 0$, respectively, for $\alpha=2$ (left), which is symmetrical (positive and negative fluctuations have same amplitudes), and $\alpha=1.2$ (right), which is extremely asymmetrical with huge negative fluctuations, but moderate positive fluctuations. Both have a fast probability falloff for positive extremes, as required.

(1) the extremely asymmetric Lévy white noise $\gamma_{0}^{(\alpha)}$ of stability index $\alpha$, often called sub-generator (Fig. 4)

(2) the generator, $\Gamma_{\lambda}$, obtained by fractional integration of order $D / 2 \alpha^{\prime}$ of the sub generator (Eqs. (34) and (35))

(3) the (normalized) flux, $\varepsilon_{\lambda}$, obtained with the help of the exponential of the generator $\Gamma_{\lambda}$ with universal parameters $C_{1}, \alpha$

(4) the multifractal field, $v_{\lambda}$, obtained by fractional integration of order $H$ of the forcing $f_{R}=\varepsilon_{\lambda}^{a}$ (Eqs. (36) and (37)).

\section{FROM PRODUCTS TO EXPONENTIAL: LIE ALGEBRA AND GROUPS}

The previous developments were inspired by discrete in scale cascades, which are obtained by products of identically independently distributed variables. However, to obtain continuous in scale processes the products were replaced by exponentials of additive processes. This rather straightforward substitution nevertheless opens the road to broad generalizations, which are needed to obtain multifractals that are
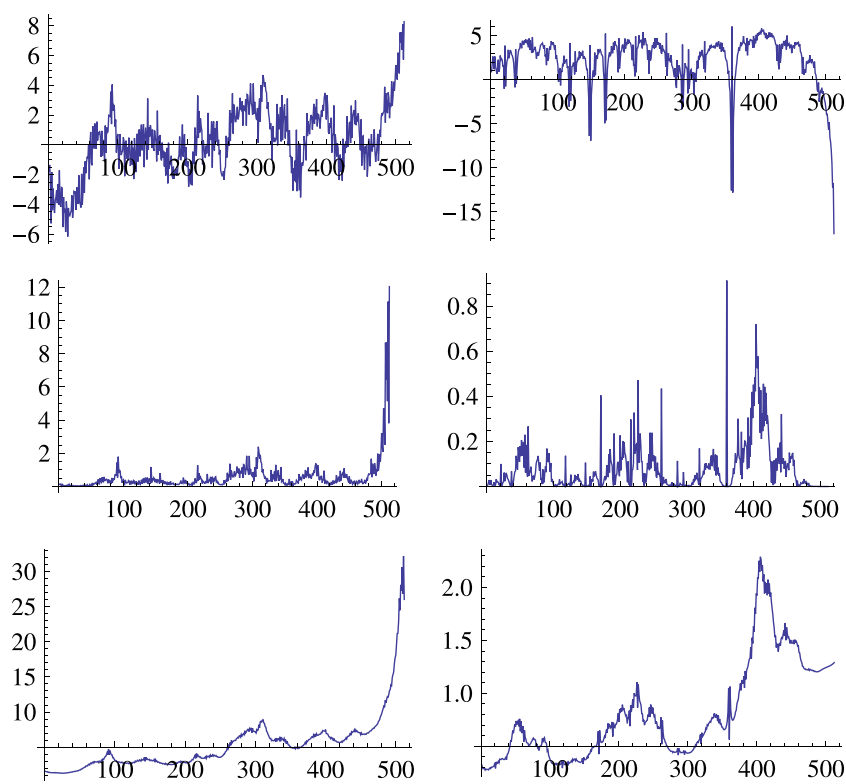

FIG. 5. Illustration of the three last steps (see text) to obtain multifractal fields from sub-generators (white noises) $\gamma_{0}^{(\alpha)}$ of Fig. 4 with parameters $C_{1}=0.2, a=1, H=1 / 9, \lambda=512$ (the horizontal axis is the time $t \in(0, \lambda)$ ), respectively, for $\alpha=2$ (left column) and $\alpha=1.2$ (right column), from top to bottom: generator $\Gamma_{\lambda}$; (conservative) flux $\varepsilon_{\lambda}$; multifractal field $v_{\lambda}$ obtained by a fractional integration of $\varepsilon_{\lambda}$. 
vector or manifold valued. This can be seen at first with the help of the matrix representations of linear operators, because the exponential of a matrix $X$ is merely defined with the help of the same expansion series as for the exponential of a scalar

$$
\exp (X)=\sum_{i=0}^{\infty} \frac{X^{n}}{n !}
$$

However, this infinite expansion series is rather formal for any $n \times n$ matrix $^{56}$ due to the existence of the minimal polynomial $\mu_{X}$, i.e., the polynomial of least degree whose $\mathrm{X}$ is a root $\left(\mu_{X}(X)=0\right)$ and whose degree $m$ is less or equal to $n$. The upper-bound $n$ is reached when the minimal polynomial corresponds to the Cayley-Hamilton characteristic polynomial. The fact that the remainders $r_{k}(x)$ of the Euclidean division of $x^{k} / k$ ! by $\mu_{X}(x)$ are all of degree strictly less than $m$ implies that the matrix exponential reduces to a polynomial of same degree

$$
\exp (X)=\sum_{i=0}^{\infty} r_{k}(X)=\sum_{i=0}^{m-1} a_{k} X^{k}
$$

The matrix exponential maps the algebra $M(n, R)$ of the real square matrices of dimension $n$ into the general linear group $G L(n, R)$ of nonsingular matrices, generalizing the mapping from the additive group $R$ into the multiplicative group $R^{+}$of positive real numbers. This corresponds to a particular case of the general concept of mapping a Lie algebra into an associated Lie group, ${ }^{57}$ at least locally (a global mapping requires the group to be simply connected). In a general manner, the group is only a smooth manifold whereas the algebra is a tangent vector space and has therefore a simpler structure. It means that differential calculus applies, e.g., vectors of the algebra can be understood as linear differential operators with respect to the local coordinates/parameters $\theta_{i}$ of the group ${ }^{58}$

$$
X=\sum_{i} X^{i} \frac{\partial}{\partial \theta^{i}}
$$

The integration of this vector field generates the group as a flow over a manifold. The infinitesimal group generator corresponds to the tangent space to the group at the identity of this group and has therefore a Lie algebra structure. This means that this vector space is endowed with a multiplication defined by a Lie bracket, which is bilinear and satisfies the Jacobi identity

$$
[X,[Y, Z]]+[Y,[Z, X]]+[Z,[X, Y]]=0
$$

and the alternating property

$$
[X, X]=0 .
$$

Both properties imply together that the Lie bracket is anticommutative

$$
[Y, X]=-[X, Y]
$$

For matrices or linear operators (e.g., linear differential operators discussed above, Eq. (40)), the Lie bracket is merely the commutator (respectively for multiplication and composition)

$$
[X, Y]=X Y-Y X
$$

whereas the anti-commutator $\{.,$.$\} is defined by$

$$
\{X, Y\}=X Y+Y X
$$

The commutator indeed satisfies the aforementioned properties (Eqs. (41)-(43)) and this corresponds to the origin of the theory. Finally, let us recall that in general

$$
\exp (X+Y) \neq \exp (X) \exp (Y)
$$

nevertheless

$$
[X, Y]=0 \Rightarrow \exp (X+Y)=\exp (X) \exp (Y),
$$

although this is only a sufficient condition.

\section{THE EXAMPLE OF PSEUDO-QUATERNIONS}

The example of the two-dimensional linear group $l(2, R)$, represented by two-dimensional matrices, has been often used, especially to define generators $G$ 's of deterministic scale transformations $T_{\lambda}$ of a two-dimensional domain. The dimension 2 corresponds indeed to the minimal complexity to obtain a noncommuting algebra, in opposition to the (commuting) fields of real or complex numbers, which are very special subsets of $l(2, R)$. The vectors of $l(2, R)$ have been often called quaternion-like or pseudo-quaternions ${ }^{28,59-61}$ for reasons discussed below.

At first, the (reduced) discriminant of the CayleyHamilton characteristic polynomial of $G$

$$
Q(G)=\left(\frac{\operatorname{Tr}(G)}{2}\right)^{2}-\operatorname{Det}(G)
$$

points out two different regimes for the scale transformation $T_{\lambda}=\lambda^{G}$ :

- a dominant rotation for $Q(G)<0$, due to complex eigenvalues

- a dominant stratification for $Q(G) \geq 0$, due to real eigenvalues

Figures 2 and 6 display the generalized scales $T_{\lambda}\left(B_{1}\right)$ of a spherical unit ball $B_{1}$, corresponding, respectively, to isotropy, pure stratification, dominant stratification, and dominant rotation. Below, we will further clarify this dichotomy with the help of an analytical expression of $T_{\lambda}$ (Eqs. (54) and (57)).

The Lie structure of $l(2, R)$ can be put in evidence by using the following vector basis:

$$
\begin{aligned}
& G=d 1+e I+f J+c K \\
& 1=\left[\begin{array}{ll}
1 & 0 \\
0 & 1
\end{array}\right] I=\left[\begin{array}{cc}
0 & -1 \\
1 & 0
\end{array}\right] \\
& J=\left[\begin{array}{ll}
0 & 1 \\
1 & 0
\end{array}\right] K=\left[\begin{array}{cc}
1 & 0 \\
0 & -1
\end{array}\right]
\end{aligned}
$$



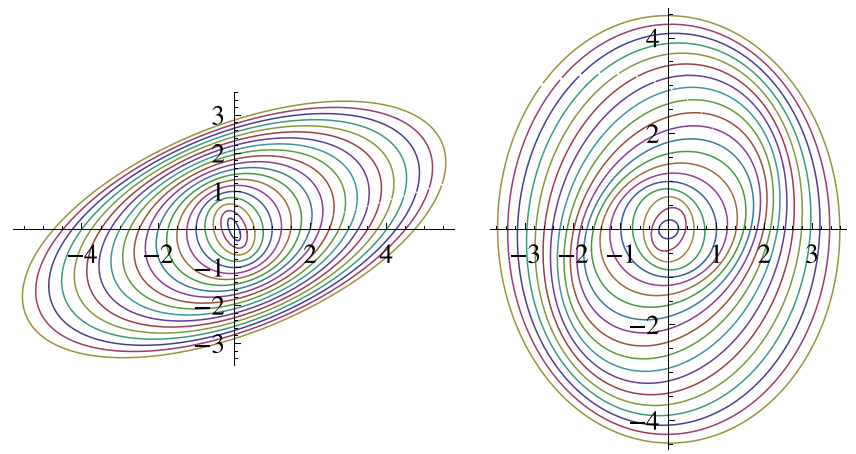

FIG. 6. Contours of $2 \mathrm{D}$ balls $B_{\lambda}$ for scale ratios $\lambda \in(1 / 4,5)$ by steps of $\delta(1 / \lambda)=0.2$ and a pseudo-quaternion generator: (a, on the left) for a dominant stratification $(Q(G)>0)$, nevertheless with some rotation $(d=1, c=f$ $=0.2, e=0.1)$; (b, on the right) for a dominant rotation $(Q(G)<0)$, although with some stratification $(d=1, c=f=0.2, e=0.7)$.

whose vectors satisfy the following commutator relations

$$
2 I=[J, K], 2 J=[I, K], 2 K=[J, I]
$$

as well as the anti-commutator relations

$$
\{I, J\}=\{J, K\}=\{K, I\}=0
$$

and the following square roots of \pm unity:

$$
I^{2}=-J^{2}=-K^{2}=I J K=-1
$$

to be compared later on with the famous quaternions equation (Eq. (61)). Together with the anticommutation (Eq. (50)), it inclines to call the $l(2, R)$ vectors "pseudo-quaternions."28,59-61 Section VIII will demonstrate that the connection between quaternions and pseudo-quaternions is even stronger than suggested by this similarity: both are examples of Clifford algebra whose common structure yield strong, generic properties.

The example of the pseudo-quaternions $(l(2, R))$ can also be used to present the Levi decomposition of any Lie algebra into its radical and a semi-simple subalgebra

$$
l(2, R)=R 1 \oplus_{s} \operatorname{sl}(2, R)
$$

where the one-dimensional subalgebra $R 1$ is generated by the identity 1 and the field of real numbers $R$, and $\operatorname{sl}(2, R)$ is the special two-dimensional real Lie linear algebra of linear applications/matrices with zero trace and is spanned by $\{I, J, K\}$.

$R 1$ is the radical of $l(2, R)$ because it is its largest abelian ideal (in short: $[R 1, l(2, R)]=0$ ), whereas $\operatorname{sl}(2, R)$ is semi-simple because its radical is on the contrary reduced to $\{0\}$. The determination of the radical is important because it commutes with all the other symmetries. However, this might have prevented for other properties of potentially relevant Lie algebra. For instance, quaternions and Clifford algebra were mentioned in the original discussion of Lie cascade, ${ }^{62}$ but unfortunately not really explored.

The Levi decomposition of $l(2, R)$ (Eq. (53)), merely corresponds to the decomposition into a trace full component $(\langle G\rangle=d 1 \in R 1)$, which is the scalar part of $G$, and a traceless component $\left(G_{0}=G-\langle G\rangle \in \operatorname{sl}(2, R)\right)$, in fact the vector part of $G$. It is helpful to derive the following generalization of the Euler identity for complex numbers:

$$
\begin{aligned}
a & =Q(G)^{1 / 2}, \quad G_{0}=G-\langle G\rangle, \quad\langle G\rangle=d 1: \\
\lambda^{G} & =\lambda^{d} \lambda^{G_{0}}=\lambda^{d}\left(\cosh (a \log (\lambda)) 1+\sinh (a \log (\lambda)) G_{0} / a\right) .
\end{aligned}
$$

At first, it is worth to note that the fact that $\lambda^{G_{0}}$ is a polynomial of order 1 is in agreement with Eq. (39). The first stage of the derivation of Eq. (54) corresponds to the fact that the tracefull $\langle G\rangle=d 1$ component of $G$ belongs to the radical $R 1$ and therefore commutes with the traceless component $G_{0}=G-\langle G\rangle \in \operatorname{sl}(2, R)$ the product of their exponentials thus correspond to that of their sum (Eq. (47)), i.e., G. The second stage merely results from the fact that the pseudoquaternion vector basis (Eq. (49)) is, due to their anticommutation (Eq. (51)), an orthogonal basis for the CayleyHamilton characteristic polynomial discriminant

$$
G_{0}^{2}=(c K)^{2}+(f J)^{2}+(e J)^{2}=Q(G) 1
$$

therefore,

$$
Q(G)=c^{2}+f^{2}-e^{2} .
$$

It then suffices to input this relation into the exponential expansion (38) to obtain Eq. (54). The hyperbolic cosine and sine becomes trigonometric cosine and sine as soon as $a=Q(G)^{1 / 2}$ becomes imaginary, i.e., $Q(G)<0$

$$
\begin{aligned}
& i a^{\prime}=Q(G)^{1 / 2}, \quad G_{0}=G-\langle G\rangle, \quad\langle G\rangle=d 1: \\
& \lambda^{G}=\lambda^{d} \lambda^{G_{0}}=\lambda^{d}\left(\cos \left(a^{\prime} \log (\lambda)\right) 1+\sin \left(a^{\prime} \log (\lambda)\right) G_{0} / a^{\prime}\right) .
\end{aligned}
$$

This obviously corresponds to a transition from a dominant stratification to a dominant rotation. We have been using until now the meteorological term "stratification" that is very suggestive for the vertical anisotropy of atmospheric dynamics, but as suggested by an anonymous referee, "stretching" or "deformation" could be more appropriate for fluid mechanics, where their relative importance with respect to rotation has been often looked for. ${ }^{63}$ Pseudoquaternions, and their generalisation discussed below, could be therefore helpful to investigate this question.

\section{CLIFFORD ALGEBRA}

Clifford algebra broadly generalize the properties that we have seen for the pseudo-quaternions, particularly the key role of the quadratic form $Q$ (Eq. (55)) and are therefore used in this paper to broadly generalize what was obtained with multifractals based on pseudo-quaternions. A key feature of these algebra is to be a "graded algebra," i.e., to have elements with different levels of complexity that are measured by a "grade." Associated to it, there are two straightforward mechanisms to upgrade or on the contrary downgrade them, ${ }^{64,65}$ see Fig. 7. More precisely, a Clifford algebra has scalars (grade 0), vectors (grade 1), bi-vectors (grade 2), etc., upgrading is merely obtained by the composition of linear applications or by the product of the corresponding matrices. Downgrading is obtained as soon as two components of the composition/ product are proportional: the quadratic from $Q$ then yields a 


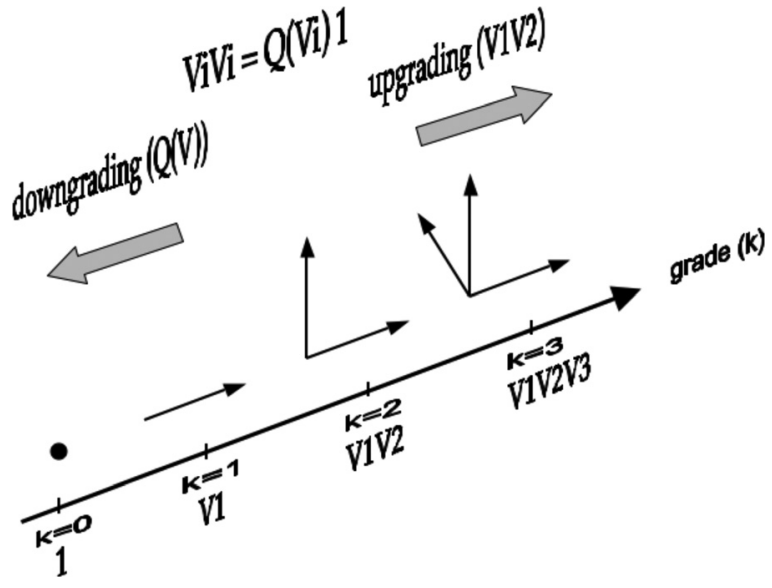

FIG. 7. Schematic of the two mechanisms of a Clifford algebra to upgrade or on the contrary downgrade its elements, respectively, with the help of their composition/multiplication and the quadratic form $Q$ : the composition/ product of components that are proportional yields only a scalar.

scalar. A Clifford algebra is therefore generated from a given vector space $V$ with the help of both mechanisms. In particular, the downgrading is merely obtained with the help of the quadratic form $Q$ that is initially defined on $V$ and generalizes that of the pseudo-quaternions (Eq. (55))

$$
v^{2}=Q(v) 1, \quad \forall v \in V
$$

Equation (56) is straightforwardly generalized into

$$
Q(v)=v_{1}^{2}+\cdots+v_{p}^{2}-v_{p+1}^{2}-\cdots-v_{p+q}^{2}
$$

simply because every nondegenerate quadratic form on a real vector space $V$ of finite dimension $n$ can be put under the canonical diagonal form with respect to an orthogonal basis; $v_{i}$ are the coordinates of $v$ with respect to this basis, the pair $(p, q)$ is the signature of the quadratic form, with $p+q=n$. The corresponding Clifford algebra is denoted $C l_{p, q}(R)$. A canonical orthogonal basis of $V$ corresponds to have $n$ mutually orthogonal vectors, $p$ of them have norm +1 and $q$ have norm -1 , and therefore $C l_{p, q}(R)$ has $p$ vectors that square to +1 and $q$ that square to -1 .

It is worthwhile to note that $C l_{0,0}(R)$ is isomorphic to $R$ ( $V=\varnothing$, no vector, only scalars), $C l_{0,1}(R)$ to $C$ (a unique vector $I$, which squares to $1, V=R I), C l_{1,0}(R)$ seems to be nonclassical (with $V=\{R J\}$ or $V=\{R K\}$ ), $C l_{2,0}(R)$ $=C l_{1,1}(R)$ corresponds to the pseudo-quaternions $l(2, R)$ (with $V=\{R J, R K\}$ for $C l_{2,0}(R), V=\{R J, R I\}$ or $V=\{R K, R I\}$ for $\left.C l_{1,1}(R)\right)$. $C l_{0,2}(R)$ corresponds to the quaternions $H$ (spanned by $1, I_{2}, J_{2}, K_{2}=I_{2} J_{2}$, whose last three elements square to -1 and anti-commute). They have the following matrice representation with block matrices $(2 \times 2)$

$$
I_{2}=\left[\begin{array}{cc}
0 & -1 \\
1 & 0
\end{array}\right] ; \quad J_{2}=\left[\begin{array}{cc}
0 & -K \\
K & 0
\end{array}\right] ; \quad K_{2}=\left[\begin{array}{cc}
-I & 0 \\
0 & I
\end{array}\right]
$$

which satisfies the famous equations

$$
I_{2}=J_{2}=K_{2}=I_{2} J_{2} K_{2}=-1 \text {. }
$$

The quaternions $H$ in turn can be generalized into the octonions $O=\mathrm{Cl}_{0,3}(R)$, however, although there is no limitation on the dimension $n$ of the vector space $V$ generating algebra $C l_{0, n}(R)$, given properties are no longer supported. Indeed, according to Hurwitz's theorem, ${ }^{66} R, C, H, O$ are the only normed division algebras over the reals. In other words, other Clifford algebra, in particular, $C l_{0, n}(R)$ for $n>3$, do no longer contains inverses, whose existence makes $H$ and $O$ very close to a field, like $R$ and $C$. In fact $H$ and $O$ lack only the commutativity of the product to be fields.

The downgrading role of the quadratic form can be better understood with the help of the corresponding ideal $I Q$

$$
I Q=\{v \in V \mid v \otimes v-Q(v) 1\}
$$

because the corresponding Clifford algebra $\operatorname{Cl}(V, Q)$ is then isomorphic to the quotient of the tensor algebra $T(V)$ generated by the vector space $V$ by the equivalence relation introduced by $I Q$

$$
C l(V, Q) \cong T(V) / I Q
$$

$T(V)$ corresponds to the opportunistic upgrading mechanism, which is limited by the downgrading quotient $I Q$. There are many abstract properties that can be deduced from this morphism, such as the fact that Clifford algebra are super algebra or have a $Z / 2 Z$ grading, which corresponds to the fact that they can be split into an even and an odd parts

$$
C l(V, Q)=C l^{0}(V, Q) \oplus C l^{1}(V, Q),
$$

with the following morphisms for real algebra:

$$
\begin{array}{ll}
C l_{p, q}^{0}(R) \cong C l_{p, q-1}(R) & \text { for } q>0, \\
C l_{p, q}^{0}(R) \cong C l_{q, p-1}(R) & \text { for } p>0 .
\end{array}
$$

This ensures a series of inclusions of Clifford Algebra, e.g.,

$$
R \subset C \subset H \subset O
$$

However, the most important consequence for us of Eq. (59) or Eq. (63) is that the derivation of Eq. (54) (or Eq. (57)) remains unchanged for any Clifford algebra when taking the appropriate tracefull/scalar component $\langle G\rangle=d 1$ and traceless component $G-\langle G\rangle$ of $G$. As final, general remarks on Clifford algebra, let $e_{1}, e_{2}, . ., e_{n}$ be an orthogonal basis of $(V, Q)$ of (finite) dimension $n$, then $C l(V, Q)$ admits the basis

$$
\left\{e_{i_{1}} e_{i_{2}} \ldots e_{i_{k}} \mid 1 \leq i_{1}<i_{2}<\cdots<i_{k} \leq n \text { and } 0 \leq k \leq n\right\}
$$

the empty product $(k=0)$ corresponds to the multiplicative unity. The dimension of $\operatorname{Cl}(V, Q)$ is therefore

$$
\operatorname{dim}[C l(V, Q)]=\sum_{k=0}^{n}\left(\begin{array}{l}
n \\
k
\end{array}\right)=2^{n}
$$

because for each $k$ there are $k$ chosen basis vectors among the $n$ basis vectors of $V$. Let us mention that the quadratic form $Q$, initially defined over $V$, can be extended over $C l(V, Q)$ with the property that 


$$
\forall v_{i} \in V: Q\left(v_{1} v_{2} \ldots v_{k}\right)=Q\left(v_{1}\right) Q\left(v_{2}\right) \ldots Q\left(v_{k}\right)
$$

but this identity is not true for arbitrary elements of $C l(V, Q)$.

\section{CLIFFORD ALGEBRA AND UNIVERSAL MULTIFRACTALS}

The obtained results, especially Eq. (54) and Eq. (57), are immediately applicable for deterministic scale transform $T_{\lambda}$ and $\tilde{T}_{\lambda}$, therefore for the resulting scaling symmetries $S_{\lambda}$ of fractal fields (Eq. (15)). Once again, they generalize to higher dimensions what we have observed for the pseudoquaternions, e.g., whether we have a dominant stratification (Eq. (54)) or a dominant rotation (Eq. (57)), see Fig. 6. However, it is worthwhile to emphasize that the use of the exponential of $C l_{0, n}$, i.e., exponentials of linear combination of square roots $L_{i}$ of minus unity,

$$
M\left(\theta_{1}, \theta_{2} \ldots, \theta_{n}\right)=\exp \left(\theta_{1} L_{1}+\theta_{2} L_{2}+\ldots+\theta_{n} L_{n}\right),
$$

have several advantages compared to the classical representation of $S O(n)$ with the help of Euler angles, i.e., the product of rotations around orthogonal axes

$$
R\left(\theta_{1}, \theta_{2} \ldots, \theta_{n}\right)=R_{x_{1}}\left(\theta_{1}\right) R_{X_{2}}\left(\theta_{2}\right) \ldots R_{x_{n}}\left(\theta_{n}\right)
$$

where $R_{x_{i}}\left(\theta_{i}\right)$ is a rotation of angle $\left(\theta_{i}\right)$ with respect to the $x_{i-}$ axis. The case for the quaternions $\left(\mathrm{Cl}_{0,2}\right)$ with respect to a $3 \mathrm{D}$ rotation matrix $(\in S O(3))$ is rather well-known, ${ }^{67}$ in particular, quaternions are immune to the gimbal lock phenomenon, whereas the latter has drastic consequences for gyroscopes and flight altitude indicators. This seems even stronger for higher dimensions. The fundamental reason is that Euler angles are not canonical coordinates, which are a system $\left\{\theta_{1}, \theta_{2} \ldots, \theta_{n}\right\}$ such that each $\theta_{i}(t)=a_{i} t$, where $a_{i}$ is a given constant, defines a one-parameter group. Whereas it is obviously the case for $M\left(\theta_{1}, \theta_{2} \ldots, \theta_{n}\right)$ (Eq. (70)), but not for $R\left(\theta_{1}, \theta_{2} \ldots, \theta_{n}\right)$ (Eq. (71)) and for the product of exponentials

$$
P\left(\theta_{1}, \theta_{2} \ldots, \theta_{n}\right)=\exp \left(\theta_{1} L_{1}\right) \exp \left(\theta_{2} L_{2}\right) \ldots \exp \left(\theta_{n} L_{n}\right) .
$$

The gimbal lock phenomenon merely results from the presence of singularities in Eq. (71).

The dichotomy between dominant rotation and dominant stratification regimes has much stronger consequences for stochastic scale transform generators than for deterministic ones. The reason is that, similarly to the scalar case, the exponential of too large fluctuations may prevent the existence of any finite statistical moment. There is not such a risk in the case of a dominant rotation due to the imaginary eigenvalues and the resulting trigonometric sine and cosine in Eq. (57) that are bounded. On the contrary, the presence of hyperbolic sine and cosine in Eq. (54) requires the generator fluctuations to respect some constraints. A slight generalization of Eq. (31) corresponds to

$$
\forall n \in N, \forall X \in R: 2 \cosh (X) \geq \exp (|X|) \geq|X|^{n} / n !,
$$

which shows that, when applied to $X=Q\left(\Gamma_{\lambda}\right)^{1 / 2} \in R$, finite statistics of the stochastic codomain scale transform $\tilde{T}_{\lambda}$ require that $\Gamma_{\lambda}$ do not have large fluctuations, i.e., a power law probability distribution tail, both for negative and positive extremes. With respect to stable Lévy generators, this constraint can be satisfied in various cases where the scalar part $\langle G\rangle$ is supposed to be independent of the vector part $G-\langle G\rangle$. They include:

- the signature of the quadratic form $Q$ is purely negative over the Clifford algebra

- the stable Lévy white noise is Gaussian, i.e., $\alpha=2$, the signature of $Q$ is no longer relevant

However, these are only particular examples.

\section{CLIFFORD LAPLACE TRANSFORM AND FINITE STATISTICS}

A systematic approach corresponds to consider the general characteristic function of a Lévy vector for a given set of statistical orders that are now vectors of the space $C l_{p, q}$. This first requires the definition of a scalar product. This is obtained with the help of the polarization identity

$$
\langle X, Y\rangle=\frac{1}{2}(Q(X+Y)-Q(X)-q(Y)) .
$$

The $q$ th moment of the codomain scale transform $\tilde{T}_{\lambda}$ for a vector $q \in C l_{p, q}$ are then defined to be the Clifford-Laplace transform of the probability of the generator. The symmetric bilinear form (Eq. (74)) can be used to define a Clifford Fourier transform, ${ }^{68}$ which has been used to some extent in signal processing. ${ }^{69}$ However, like for the scalar case (Eq. (20)), we are interested by a Clifford Laplace transform of the probability of the generator $\Gamma_{\lambda}$ that corresponds to the scalar statistical moments of $\tilde{T}_{\lambda}$

$$
E\left[\tilde{T}_{\lambda}^{q}\right]=E\left[\exp \left(\left\langle q, \Gamma_{\lambda}\right)\right\rangle\right]=Z_{\lambda}(q)=\exp \left(K_{\lambda}(q)\right) .
$$

Like for the scalar case, all these quantities are asymptotically power laws as soon as $K_{\lambda}(q)$ has a $\log (\lambda)$ divergence. However, the finiteness of the Clifford-Laplace transform, like for the classical Laplace transform, requires some constraints. But we need first to briefly discuss the generalization of stable Lévy variables to stable Lévy vectors. Considering the linearity of the stability and attractivity of stable Lévy variables (Eqs. (22) and (23)), this generalization seemed so trivial to Lévy ${ }^{49}$ that he did not explicitly write it. Indeed, the classical generalization ${ }^{49,70,71}$ corresponds to consider that the only change in Eqs. (22) and (23) is that the centering term $b$ becomes a vector as soon as the random variables $X_{i}$ and $Y_{i}$ become random vectors, while the renormalizing factor $a(n)$ and its group generator $1 / \alpha$ (Eq. (25)) are kept scalar. Still due to the aforementioned linearity, the component $\langle X, u\rangle$ of a stable Lévy vector $X$ along any given direction $u(Q(u)=1)$ of a Lévy vector is a stable variable with the same stability index and of the same type, e.g., symmetric or strictly stable. This is not only a necessary condition to have a stable vector but also it is sufficient for strictly stable vector or for a stability index $\alpha>1$. The second Fourier characteristic function of a Levy vector is classical, ${ }^{49,70,71}$ whereas its second Laplace characteristic function $K_{X}(q)=\log E[\exp (\langle q, X\rangle)]$ is less classical $^{42}$ 


$$
\text { if } \begin{aligned}
\partial B_{1}^{\prime}(q) \subset\{u \mid\langle q, u\rangle \geq 0\}: K_{X}(q) \\
=\int_{\partial B_{1}^{\prime}(q)}\langle q, u\rangle^{\alpha} d \Sigma_{X}^{\prime}(u)+\langle q, b\rangle ; \\
\text { otherwise }: K_{X}(q)=\infty,
\end{aligned}
$$

where $\partial B_{1}^{\prime}(q) \subset \partial B_{1}$ is the support of the spectral measure $d \Sigma_{X}^{\prime}(u)$ and $\partial B_{1}=\{u \mid Q(q)=1\}$ is the surface of the unit sphere. At first, $K_{X}(q)$ defined by Eq. (76) satisfies the following scale symmetry:

$$
\forall \lambda \in R^{+}, \forall q \in R: K_{X}\left(\lambda^{1 / \alpha} q\right)=\lambda K_{X}(q)
$$

as required first for any positive integer $\lambda$ by Eq. (22), then for any inverse of integer $\lambda$, by considering the intermediate vector $q^{\prime}=-\lambda^{-1 / \alpha} q$, therefore for any rational $\lambda$. Finally, due to the continuity of $K_{X}(q)$, this is true for any positive real $\lambda$.

The asymmetry of the measure $\Sigma_{X}^{\prime}$ rules the statistical asymmetry of the vector. As already mentioned, $\alpha=2$ does not introduce any constraint on $q: \Sigma_{X}^{\prime}(u)$ and $\langle X, u\rangle$ are then symmetric. On the contrary, $\alpha<2$ requires $\langle X, u\rangle$ to be extremely asymmetric (i.e., no heavy tail for positive extremes) and $\langle q, u\rangle \geq 0$ is a generalization of scalar constraint $q \geq 0$. Without entering into the technical details, let us mention that the measure $d \Sigma_{X}^{\prime}(u)$ (Eq. (76)) is discrete if and only if the stable Lévy vector can be expressed as a linear transform of independent stable Lévy random variables. On the contrary, cross-dependence between components can be analyzed with the help of copulas. ${ }^{72}$ The measure $\Sigma_{X}^{\prime}(u)$ is in fact defined with the help of the Lévy canonical measure $F_{Y}(u)$ that distributes the jumps $Y$ along the direction $u$, in fact in the opposite direction to $u$, because extremely asymmetric $\langle X, u\rangle$ with only negative heavy tails are obtained when large jumps are distributed in the direction opposite to $u$.

At first glance, the support $\partial B_{1}^{\prime}$ seems to be a bit complex because it involves the two independent vectors $q$ and $u$. However, if we furthermore require that the one-parameter groups $\exp \left(\theta_{i} L_{i}\right)$ (Eq. (70)) have finite statistical moments $E\left[\exp \left(\left\langle q, L_{i}\right\rangle\right)\right]$ and therefore finite $K_{X}(q)$ for $q$ along a basis vector $L_{i}$, the support $\partial B_{1}^{\prime}$ belongs to the intersection of the unit sphere surface $\partial B_{1}$ with that of the half spaces $\left\langle L_{i}, u\right\rangle \geq 0$, i.e., with the hyper-cube $C=\left\{u_{i} \geq 0\right\}$.

This is an important result; however, the classical definition of stable Lévy vector used so far is not general enough and rather restrictive, because all the vector components have the same Levy stability index $\alpha$. This definition was indeed called quasi-scalar ${ }^{42}$ and it was suggested to consider a wider definition, still based on the linearity of definitions of stability and attractivity (Eqs. (22) and (23)), but where both the renormalizing factor $a(n)$ and its group generator $1 / \alpha$ (Eq. (25)) are matrices. The direction dependence of $\alpha$ obviously introduces a much stronger anisotropy than that of the spectral measure $\Sigma_{X}^{\prime}$. Although the fundamental scale symmetry (Eq. (77)) of the characteristic function $K_{X}(q)$ remains rather unchanged, the matrix nature of $\alpha$ introduces a foreseen complexity in the measure $\Sigma_{X}^{\prime}(u)$, in fact in the more fundamental Lévy canonical measure $F_{Y}(u)$. This results from the fact that the spectrum $\operatorname{Spec}(\alpha)=\left\{\nu_{i}\right\} \subset(0,2]$ of $\alpha$ is no longer reduced to a unique eigenvalue. While the constraint $\langle q, u\rangle \geq 0$ remains the same for eigenspaces with $\nu_{i}<2$, it is no longer relevant for those with $\nu_{i}=2$.

The above results enable us to obtained Lévy whitenoises $\gamma_{0}^{(\alpha)}$ valued on a given Clifford algebra $C l_{p, q}$ for a given stability index $\alpha$ that is either a (unique) scalar or a matrix. We can therefore follow the same four steps discussed in Sec. VC and illustrated by Figs. 4 and 5, transposing mutatis mutandis the scalar equations into equations on $\mathrm{Cl}_{p, q}$. One may note that:

- if $\alpha$ remains a scalar, propagators $G$ and $G_{R}$, respectively, defined by Eq. (35) can be preserved as are, i.e., to be scalars

- $\alpha$ is no longer a scalar, one needs to define $-\left(-\Delta_{x}\right)^{M}$, $M \in C l_{p, q}$, as the inverse Clifford-Fourier transform of $\|k\|^{2 M}=\exp \left(2 M \log (\|k\| \mid)\right.$ for $G$ and $G_{R}$

- the codomain scale transform $\tilde{T}_{\lambda}$ is obtained with the help of the generalized Euler identity (Eq. (54)) applied to the generator $\Gamma_{\lambda}$ instead of its exponentiation

- the flux $\varepsilon_{\lambda}$ is a vector on $R^{D}$ and is obtained by applying $\tilde{T}_{\lambda}$ to the homogeneous (large scale) field $\varepsilon_{1}$.

Figures 8 and 9 display snapshots of two numerical simulations of this type, with scalar exponents $\alpha=1.5, H$ $=1 / 3, a=1 / 3$. They correspond, respectively, to:

- a $2 \mathrm{D}+1$ multifractal complex velocity field, i.e., the domain is $R^{3}$ and the codomain is $C=C l(0,1)$. The arrows represent the (horizontal) velocity

- a 3D + 1 multifractal quaternion velocity field, i.e., the domain is $R^{4}$ and the codomain is $Q=C l(0,2)$ whose only the first three components are represented by the 3D arrows.

In both cases, the initial condition was taken as a uniform flow along the $\mathrm{x}$-axis, therefore a similar condition for the flux energy: $\varepsilon_{1}=1$. The numerical convolutions were

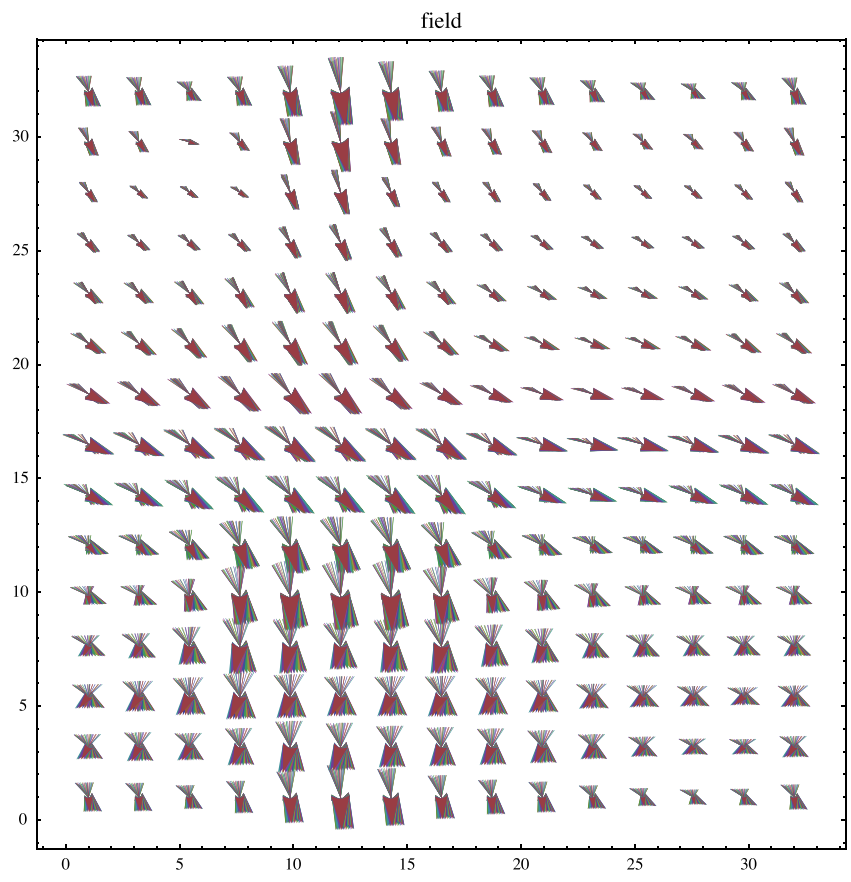

FIG. 8. Snapshot of a multifractal simulation of a $2 \mathrm{D}+1$ intermittent vector field obtained by a complex cascade, i.e., with values on $C l(0,1)$, see text for the details of the numerical simulation. 


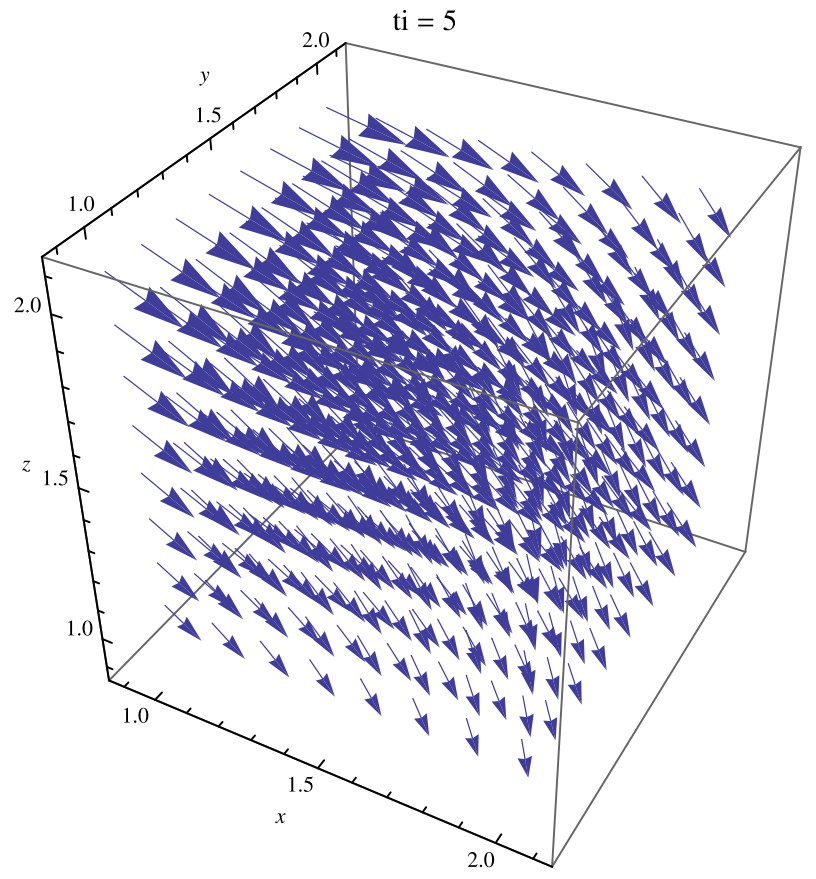

FIG. 9. Snapshot of a multifractal simulation of a $3 \mathrm{D}+1$ intermittent vector field obtained by a quaternion cascade, i.e., with values on $C l(0,2)$, see text for the details of the numerical simulation.

performed in the physical space with the help of Mathematica. The choice of quaternions was due to the fact that quaternions were already implemented; however, any other noncommutative algebra can be implemented.

\section{CONCLUSIONS}

This paper shows that the combination of stable Lévy processes with Clifford algebra defines in a precise manner a wide class of non trivial, stochastic symmetries, as well as fields respecting these symmetries that can be either vectorvalued or manifold-valued with arbitrary large dimension. It was also pointed out that there is no basic difficulty to proceed to numerical simulations of the vector fields. The range of potential applications is large because this combination relies on a few fundamental properties: the stability and attractivity of stable Lévy processes and their resulting Laplace characteristic function, Clifford algebra are Lie algebra with a quadratic form whose signature is qualitatively important, the exponential maps it into its Lie group and enables to defines statistical moments of generalized scale transforms with the help of a Clifford-Laplace transform. Overall, the fundamental structure on which this approach is based can be tentatively called a Lévy-Clifford algebra.

Because the vector structure of this stochastic algebra is much simpler that the manifold structure of the group, this should help to resolve deadlocks in a wide range of scientific problems encountered on complex systems having nontrivial symmetries and multiscale behavior. This is the case of the millennium problem of turbulence, which largely motivated this approach, as well as many other geophysical problems.

However, it should be noted that we only addressed the bare properties related to the generation of these processes, whereas multifractal transitions would be quite important to, respectively, assess the extremes (first order transition) and remove finite sample size biases from parameter estimation (second order phase transition). For instance, the analogues of critical temperature are obviously no longer scalars, but vectors of $C l(p, q)$, therefore, correspond to more complex phase transitions. Their understanding should provide a qualitative understanding of multicomponent systems.

\section{ACKNOWLEDGMENTS}

This research was partially supported by the Chair "Hydrology for Resilient Cities" endowed by Veolia (http:// www.enpc.fr/node/1073) and by the RainGain project (www.raingain.eu) of the North West Europe Interreg program (https://www.nweurope.eu). D. Schertzer is grateful to L. Kavvas for his kind invitation and generous financial support to participate to the Orlob International Symposium on Theoretical Hydrology that he organized at UC Davis on August 5-6, 2013, and during which preliminary results of this paper were presented and discussed. We acknowledge stimulating and thoughtful comments and suggestions of the anonymous referees.

${ }^{1}$ D. Schertzer and S. Lovejoy, "On the dimension of atmospheric motions," in 6th Symposium Turbulence and Diffusion, edited by T. Tatsumi (Elsevier Science Publishers B. V., Amsterdam, 1983), Vol. 5, pp. 69-72.

${ }^{2}$ R. Benzi, G. Paladin, G. Parisi, and A. Vulpiani, "On the multifractal nature of fully developed turbulence," J. Phys. A 17, 3521-3531 (1984).

${ }^{3}$ G. Parisi and U. Frisch, "On the singularity structure of fully developed turbulence," in Turbulence and Predictability in Geophysical Fluid Dynamics and Climate Dynamics, edited by M. Ghil, R. Benzi, and G. Parisi (North Holland, Amsterdam, 1985), pp. 84-88.

${ }^{4}$ T. C. Halsey, M. H. Jensen, L. P. Kadanoff, I. Procaccia, and B. Shraiman, "Fractal measures and their singularities: The characterization of strange sets," Phys. Rev. A 33, 1141-1151 (1986).

${ }^{5}$ B. B. Mandelbrot, "Random multifractals negative dimensions and the resulting limitations of the thermodynamic formalism," Proc. R. Soc. London A 434, 79-88 (1991).

${ }^{6} \mathrm{P}$. Brax and R. Pechanski, "Levy stable law description on intermittent behaviour and quark-gluon phase transitions," Phys. Lett. B 253, 225-230 (1991).

${ }^{7}$ S. P. Ratti, G. Salvadori, G. Gianini, S. Lovejoy, and D. Schertzer, "A universal multifractal approach to intermittency in high energy physics," Z. Phys. C 61, 229-237 (1994).

${ }^{8}$ K. Fraedrich and R. Blender, "Scaling of atmosphere and ocean temperature correlations in observations and climate models," Phys. Rev. Lett. 90, 108501 (2003); e-print arXiv:0305080 [physics].

${ }^{9}$ J. F. Royer, Biaou, F. Chauvin, D. Schertzer, and S. Lovejoy, "Multifractal analysis of the evolution of simulated precipitation over France in a climate scenario," C. R. Geosci. 340, 431 (2008).

${ }^{10}$ I. Bordi, K. Fraedrich, and A. Sutera, "Northern Hemisphere climate trends in reanalysis and forecast model predictions: The $500 \mathrm{hPa}$ annual means," Geophys. Res. Lett. 37, 1-4, doi:10.1029/2010GL043217 (2010).

${ }^{11}$ S. Lovejoy and D. Schertzer, The Weather and Climate: Emergent Laws and Multifractal Cascades (Cambridge University Press, Cambridge, U.K., 2013), p. 475.

${ }^{12}$ S. Ghashghaie, W. Breymann, J. Peinke, P. Talkner, Y. Dodge, and Ganshghaie, "Turbulent cascades in foreign exchange markets," Nature 381, 767-770 (1996).

${ }^{13}$ F. Schmitt, D. Schertzer, and S. Lovejoy, "Multifractal fluctuations in finance," Int. J. Theor. Appl. Finance 3, 361-364 (2000).

${ }^{14}$ F. S. Labini and L. Pietronero, "Statistical properties of galaxy distributions," Nonlinear Processes Geophys. 3, 274-283 (1996).

${ }^{15}$ P. Garrido, S. Lovejoy, and D. Schertzer, "Multifractal processes and selforganized-criticality in the large-scale structure of the universe," Physica A 225, 294-311 (1996).

${ }^{16}$ H. Weyl, Symmetry (Princeton University Press, Princeton, NJ, 1952).

${ }^{17}$ A. Zee, Fearful Symmetry: The Search for beauty in Modern Physics (Colliers Books, Macmillan Publishing Company, New-York, 1986).

${ }^{18}$ L. Sedov, Similitudes et Dimensions en Mécanique (MIR, Moscow, 1972). 
${ }^{19} \mathrm{E}$. Buckingham, "Physically similar systems: Illustrations of the use of dimensional equations," Phys Rev. 4, 345-376 (1914).

${ }^{20}$ E. Buckingham, "The principle of similitude," Nature 96, 396-397 (1915).

${ }^{21}$ A. A. Sonin, "A generalization of P-theorem and dimensional analysis," Proc. Natl. Acad. Sci. 101, 8525 (2004).

${ }^{22}$ M. L. Kavvas and A. Karakas, "On the stochastic theory of solute transport by unsteady and steady groundwater flow in heterogeneous aquifers," J. Hydrol. 179, 321-351 (1996).

${ }^{23}$ M. Cayar and M. L. Kavvas, "Symmetry in nonlinear hydrologic dynamics under uncertainty: Ensemble modeling of 2D Boussinesq equation for unsteady flow in heterogeneous aquifers," J. Hydrol. Eng. 14, 1173-1184 (2009).

${ }^{24}$ I. Haltas and M. L. Kavvas, "Scale invariance and self-similarity in kinematic wave overland flow in space and time," Hydrol. Processes 25, 3659-3665 (2011).

${ }^{25}$ J. Peinke, R. Friedrich, and A. Naert, "A new approach to characterize disordered structures,” Z. Naturforsch. A 52, 588-592 (1997).

${ }^{26} \mathrm{R}$. Friedrich and J. Peinke, "Description of a turbulent cascade by a Fokker-Planck equation,” Phys. Rev. Lett. 78, 863-866 (1997).

${ }^{27}$ R. Friedrich, J. Peinke, M. Sahimi, and M. R. R. Tabar, "Approaching complexity by stochastic methods: From biological systems to turbulence," Phys. Rep. 506, 87-162 (2011).

${ }^{28} \mathrm{D}$. Schertzer and S. Lovejoy, "Generalised scale invariance in turbulent phenomena," Physico-Chemical Hydrodynamics J. 6, 623-635 (1985).

${ }^{29}$ D. Schertzer and S. Lovejoy, "Physical modeling and analysis of rain and clouds by anisotropic scaling multiplicative processes," J. Geophys. Res. D 8, 9693-9714, doi:10.1029/JD092iD08p09693 (1987).

${ }^{30}$ D. Schertzer and S. Lovejoy, "Universal multifractals do exist!," J. Appl. Meteorol. 36, 1296-1303 (1997).

${ }^{31}$ H. Hagen and G. Scheuermann, "Clifford algebra and flows," Mathematical Methods for Curves and Surfaces (Springer, 2001), p. 173.

${ }^{32}$ H. Kunita, Stochastic Flows and Stochastic Differential Equations (Cambridge University Press, Cambridge, 1990).

${ }^{33}$ D. Applebaum and H. Kunita, "Lévy flows on manifolds and Lévy processes on lie group," J. Math. Kyoto Univ. 33, 1103-1123 (1993).

${ }^{34}$ B. B. Mandelbrot, The Fractal Geometry of Nature (Freeman, San Francisco, 1983).

${ }^{35}$ D. Schertzer and S. Lovejoy, "The dimension and intermittency of atmospheric dynamics," in Turbulent Shear Flow 4, edited by B. Launder (Springer-Verlag, 1985), pp. 7-33.

${ }^{36}$ Y. Chigirinskaya, D. Schertzer, S. Lovejoy, A. Lazarev, and A. Ordanovich, "Unified multifractal atmospheric dynamics tested in the tropics, part I: horizontal scaling and self organized criticality," Nonlinear Processes Geophys. 1, 105-114 (1994).

${ }^{37}$ A. Lazarev, D. Schertzer, S. Lovejoy, and Y. Chigirinskaya, "Unified multifractal atmospheric dynamics tested in the tropics: part II, vertical scaling and Generalized Scale Invariance," Nonlinear Processes Geophys. 1, 115-123 (1994).

${ }^{38}$ M. Lilley, S. Lovejoy, K. Strawbridge, and D. Schertzer, "23/9 dimensional anisotropic scaling of passive admixtures using lidar data of aerosols," Phys. Rev. E 70, 36307 (2004).

${ }^{39}$ A. Radkevich, S. Lovejoy, K. Strawbridge, and D. Schertzer, "The elliptical dimension of space-time atmospheric stratification of passive admixtures using lidar data," Physica A 382, 597-615 (2007).

${ }^{40}$ S. Lovejoy, A. F. Tuck, D. Schertzer, and S. J. Hovde, "Reinterpreting aircraft measurements in anisotropic scaling turbulence," Atmos. Chem. Phys. 9, 5007-5025 (2009).

${ }^{41}$ D. Schertzer, I. Tchiguirinskaia, S. Lovejoy, and A. F. Tuck, "Quasi-geostrophic turbulence and generalized scale invariance, a theoretical reply," Atmos. Chem. Phys. 12, 327-336 (2012).

${ }^{42}$ D. Schertzer, M. Larcheveque, J. Duan, and S. Lovejoy, "Generalized stable multivariate distribution and anisotropic dilations," IMA preprint, Minnesota U. 2, 1-11 (1999); e-print arXiv:9912016 [chao-dyn].

${ }^{43}$ B. Mandelbrot, "Self-affine fractal sets, I: The basic fractal dimensions," in Fractals in Physics, edited by L. Pietronero and E. Tosatti (NorthHolland, Amsterdam, 1985), pp. 3-16.
${ }^{44}$ J. H. Shapiro, Composition Operators and Classical Function Theory (Springer-Verlag, New York, 1993).

${ }^{45}$ J. Lamperti, "Semi-stable stochastic processes," Trans. Am. Math. Soc. 104, 62-78 (1962).

${ }^{46}$ H. E. Hurst, "Long-term storage capacity of reservoirs," Trans. Am. Soc. Civil Eng. 116, 770-808 (1951).

${ }^{47}$ V. Klemes, “The Hurst phenomenon: A puzzle?," Water Resour. Res. 10, 657-688 (1974).

${ }^{48}$ B. B. Mandelbrot and J. W. Van Ness, "Fractional Brownian motions, fractional noises and applications," SIAM Rev. 10, 422-450 (1968).

${ }^{49} \mathrm{P}$. Lévy, Théorie de l'addition des variables aléatoires (Gauthiers Villars, Paris, 1937).

${ }^{50}$ B. V. Gnedenko and A. N. Kolmogorov, Limit Distribution for Sums of Independent Random Variables (Addison-Wesley, 1954).

${ }^{51} \mathrm{~W}$. Feller, An Introduction to Probability Theory and its Applications (Wiley, New York, 1971), Vol. 2.

${ }^{52} \mathrm{~V}$. Zolotarev, One-Dimensional Stable Distributions (American Mathematical Society, Providence RI, 1986).

${ }^{53}$ A. H. Fan, "Chaos additif et multiplicatif de Levy," C. R. Acad. Sci. Paris Seie 308(5), 151-154 (1989).

${ }^{54}$ M. Metivier, Semimartingales: A Course on Stochastic Processes (Walter de Gruyter, Berlin, New York, 1982), p. 287.

${ }^{55}$ D. Schertzer, S. Lovejoy, F. Schmitt, I. Tchiguirinskaia, and D. Marsan, "Multifractal cascade dynamics and turbulent intermittency," Fractals 5, 427-471 (1997)

${ }^{56} \mathrm{H}$.-W. Cheng and S. S. Yau, "More explicit formulas for the matrix exponential," Linear Algebra Its Appl. 262, 131-163 (1997).

${ }^{57}$ D. H. Sattinger and O. L. Weaver, Lie Groups and Algebras with Applications to Physics, Geometry and Mechanics (Springer-Verlag, NewYork, Berlin, 1986).

${ }^{58}$ Gilmore, Lie Groups (Wiley, New York, 1941).

${ }^{59}$ D. Schertzer and S. Lovejoy, "Multifractal simulations and analysis of clouds by multiplicative processes," Atmos. Res. 21, 337-361 (1988).

${ }^{60} \mathrm{D}$. Schertzer and S. Lovejoy, "Nonlinear variability in geophysics: Multifractal analysis and simulation," in Fractals: Physical Origin and Consequences, edited by L. Pietronero (Plenum, New-York, 1989), p. 49.

${ }^{61}$ J. Kocik, "Clifford algebras and Euclid's parametrization of pythagorean triples," Adv. Appl. Clifford Algebras 17, 71-93 (2007).

${ }^{62}$ D. Schertzer and S. Lovejoy, "From scalar cascades to Lie cascades: Joint multifractal analysis of rain and cloud processes," in Spaceltime Variability and Interdependance for Various Hydrological Processes, edited by R. A. Feddes (Cambridge University Press, Cambridge, UK, 1995), pp. 153-173.

${ }^{63}$ J. Weiss, "The dynamic of enstrophy transfer in two-dimensional hydrodynamics," Physica D 48, 273-294 (1991).

${ }^{64}$ W. E. Baylis, "Applications of Clifford Algebras in Physics," in Lectures on Clifford (Geometric) Algebras and Applications, edited by $\mathrm{R}$. Ablamowicz and G. Sobczyk (Springer, 2004), pp. 91-133.

${ }^{65}$ P. Anglès, "The structure of the Clifford algebra," Adv. Appl. Clifford Algebras 19, 585-610 (2009).

${ }^{66}$ A. Hurwitz, "Über die Komposition der quadratischen Formen," Math. Ann. 88, 1-25 (1922).

${ }^{67}$ J. B. Kuipers, Quaternions and Rotation Sequences: A Primer with Applications to Orbits, Aerospace, and Virtual Reality (Princeton University Press, 1999).

${ }^{68}$ J. Ebling and G. Scheuermann, "Clifford Fourier transform on vector fields," IEEE Trans. Visualization Comput. Graphics 11, 469-479 (2005).

${ }^{69}$ E. M. S. Hitzer and B. Mawardi, "Clifford Fourier transform on multivector fields and uncertainty principles for dimensions $\mathrm{n}=2(\bmod 4)$ and $\mathrm{n}=3(\bmod 4)$," Adv. Appl. Clifford Algebras 18, 715-736 (2008).

${ }^{70}$ V. J. Paulauskas, "Some remarks on Multivariate Stable Distributions," J. Multivar. Anal. 6, 356-368 (1976).

${ }^{71}$ C. L. Nikias and M. Shao, Signal Processing with Alpha-Stable Distributions and Applications (John Wiley and Sons, New-York, 1995).

${ }^{72}$ J. Kallsen and P. Tankov, "Characterization of dependence of multidimensional Levy processes using Levy copulas," J. Multivar. Anal. 97, 1551-1572 (2006). 